\title{
Propuesta de valoración técnico-táctica mediante una situación de juego colectivo básico en el fútbol de iniciación

\author{
Proposal of valuation tactical-technique by means of a situation of basic collective game
} in theinitiation soccer
}

\author{
Gustavo Vera Haro*, José Pino Ortega**, Cipriano Romero Cerezo*** y María Isabel Moreno Contreras** \\ *C.E.I.P. San José Palenciana (Córdoba), **Universidad de Extremadura, ***Universidad de Granada
}

Resumen: El objetivo que buscamos con este trabajo es el de ofrecer a los técnicos de fútbol base una herramienta que les permita cuantificar el rendimiento técnico-táctico de sus jugadores y así saber en que sentido han de mejorar los mismos o hacia donde ha de focalizar los procesos de enseñanza-aprendizaje.Desde hace unos años se viene desarrollando una amplia discusión científica entre los partidarios de una iniciación deportiva centrada en el conocimiento táctico del juego y aquellos otros que optan por una visión más mecanicista y consideran oportuno comenzar con la enseñanza de la técnica, para después ir abordando conceptos tácticos. La técnica no tiene ningún sentido sin la táctica, pero ésta no podrá ver cumplidos sus objetivos sin la ayuda de aquella.

Palabras clave: Fútbol, cuantificación, técnico-táctica, enseñanza, aprendizaje.

\begin{abstract}
Our main objective is to provide minor league soccer coaches of a tool to quantify the technical-tactical performance of their players both for assessment and further planning.The question if the teachhing of games should be firstly based on the development of the tactical awareness ratther than the mechanical aspects of the skills has been running for several years on. Definitively, technic makes no sense without tactics but tactical objectives are harder made without sound tecnical foundations.
\end{abstract}

Key words: Soccer, quantification, technical-tactical, education, learning.

\section{Introducción.}

La mayoría de los trabajos que se han realizado en torno a la actuación de los jugadores, podemos comprobar como la mayoría se centran en el análisis de la dimensión técnica, no prestando mucha atención al comportamiento táctico de los mismos. Es decir, se redunda una y otra vez en la importancia que desde siempre se le ha otorgado al componente ejecutivo de las habilidades, obviando en muchos casos los procesos perceptivos y decisionales de los jugadores.

Los análisis que tradicionalmente han realizado los técnicos prestaban mucha atención a los balones perdidos, balones tocados, faltas realizadas, interceptaciones...., pero siempre se centraban en el aspecto puramente técnico, por lo cual se dejaban olvidados los aspectos más importantes del juego. En este sentido, un jugador podía realizar 25 pases correctos de 25 intentos, pero no se atendía a si las opciones elegidas por el jugador eran las correctas o no, si ese pase beneficiaba el juego o no, en definitivas cuantas, que independientemente de que la ejecución de la habilidad fuera correcta, nos podríamos encontrar con que la decisión tomada no era la más idónea.

Ante esta coyuntura, creemos conveniente encontrar una manera de hacer ver a los técnicos cual está siendo realmente la actuación de su jugador, no desde el punto de vista técnico, ni simplemente del táctico, sino desde el punto de vista técnico-táctico.

Para ello pretendemos elaborar un índice de actuación técnicotáctica a través del análisis de la actuación del jugador.

\subsection{El entrenamiento del fútbol como proceso didáctico.}

El entrenamiento del fútbol no se puede entender de una manera completa sino tenemos en cuenta sus diversas facetas, biológica, deportiva, técnico-táctica, psicológica y afectiva-social. En este trabajo nos vamos a centrar en el aspecto didáctico del entrenamiento, prestando especial atención a su aspecto cognoscitivo.

El objetivo último del entrenamiento es construir un proceso estructurado racionalmente para aumentar las prestaciones o eficacia del

Fecha de recepción: 30-05-07 - Fecha de aceptación: 30-06-07

Correspondencia: José Pino Ortega

Avenida de la Univesida

10071 Cáceres

E-mail: jpino@unex.es individuo a un nivel físico, técnico/táctico, psíquico, cognitivo y socioafectivo en un determinado deporte (Romero, 2004). Para aumentar dichas prestaciones se hace inevitable conocer cuales son las que posee el deportista y, evidentemente, cual es el nivel de desarrollo de las mismas, puesto que sin dicho conocimiento resultará imposible plantear el proceso de mejora del cual hemos hablado en la definición anterior.

Tradicionalmente, las preocupaciones del entrenador se han centrado en el conocimiento de los factores técnicos, tácticos y físicos del deportista, haciéndolo de manera aislada y sin entenderlos como un todo que influye en el rendimiento final de aquel. La iniciación al fútbol no ha permanecido ajena a esta creencia y se ha dado, por encima de todo, el desarrollo de factores técnicos y físicos en primer lugar, para posteriormente afrontar aspectos tácticos, pero, como hemos dicho con anterioridad, de manera aislada con respecto a los otros factores del entrenamiento.

Para solventar estas circunstancias nos vamos a centrar en el entrenamiento desde un punto de vista más pedagógico y didáctico. El entrenamiento es una acción pedagógica compleja, que tiene una organización específica, lo que le convierte en una acción sistemática, compleja y global sobre la personalidad y sobre el estado físico, técnico, táctico y socioafectivo del sujeto. (Romero, 2004)

Para saber si se consiguen los aprendizajes deseados, es necesario comparar los resultados con relación a los rendimientos iniciales y los objetivos que se pretendían. Esta evaluación de los resultados es en verdad quien nos dice que las actividades propuestas, la forma de realización y organización, son adecuados o no para conseguir los objetivos o resultados del entrenamiento (aprendizajes futbolísticos y personales). Para tal fin, nos vamos a centrar en Grosser, Brüggemann y Zintl (1989).

Dadas las exigencias que tiene el entrenamiento, es necesaria una orientación didáctica para sistematizar y controlar los aprendizajes y los esfuerzos que se ponen para conseguirlos.

$\mathrm{Si}$ analizamos los entrenamientos seguidos por una parte importante de los equipos de fútbol en las categorías de iniciación, podremos comprobar como en muchos casos, la construcción y diseño de los mismos deja mucho que desear en cuanto a coherencia, sistematicidad, adecuación a las características de los jugadores, etc., pero donde creemos que existe una falta total de aplicación es precisamente en el proceso evaluativo de dicho entrenamiento, pues tradicionalmente y aún en la 
actualidad se toma como referencia normativa el rendimiento de los deportistas en competición, considerando a éste como único indicador fiable del proceso de aprendizaje del jugador. No queremos decir que tomar la competición como un lugar idónea para la evaluación no sea una práctica adecuada, si bien si entendemos que tomarla como única referencia si lo constituye. Ahora bien, el principal problema lo encontramos en la manera de afrontar dicha evaluación, ya que en la práctica totalidad de las ocasiones responde a criterios subjetivos del técnico, faltos de cualquier sistematicidad y objetividad y limitados a la capacidad de observación de éste en situación real de juego.

Bajo esta coyuntura, los juicios ${ }^{1}$ que podrá emitir el técnico acerca del aprendizaje de sus jugadores serán extremadamente restringidos y posiblemente no incorporen mucha información de utilidad al proceso de enseñanza/entrenamiento-aprendizaje.

\subsection{Construcción de una herramienta de evaluación.}

Con el objetivo de dar respuesta a la necesidad de construir un sistema de evaluación sistemático y que proporcione al técnico información adecuada y precisa de lo que está ocurriendo en el proceso de enseñanza-aprendizaje, queremos construir una herramienta que ejemplifique una manera, entre muchas, de proceder en la evaluación técnico-táctica del jugador de fútbol en las categorías de iniciación.

La principal característica de la que queremos dotar a nuestra herramienta es que permita, ante todo, dar una información que vaya más allá del desenvolvimiento técnico del jugador y apunte de una manera concisa al conocimiento táctico del mismo y a la capacidad de éste de aplicarlo en el juego. Es decir no nos preocupa sólo lo que sabe hacer el jugador (aspecto técnico), sino si es capaz de aplicarlo con coherencia a la situación de juego en la que se encuentre (aspecto técnico-táctico). En definitivas cuentas, queremos valorar las habilidades ${ }^{2}$ técnico-tácticas del jugador.

Del mismo modo, otro de los aspectos que queremos paliar con la construcción de esta herramienta es la procedencia del conocimiento del técnico. Convendría cuestionarse cual es dicha procedencia, si son conocimientos ordinarios, auspiciados por la práctica, por la experiencia, por la intuición o por el parecer subjetivo de los mismos, o por el contrario son conocimientos científicos, apoyados en experiencias sistemáticas, elaboradas para obtener información válida, fiable y útil para la construcción o diseño del entrenamiento.

El técnico o entrenador deberá tener las soluciones para dar respuesta a las diversas situaciones que se pueda encontrar. Es un conocimiento práctico que, apoyado en una base cultural del entrenamiento deportivo (biológicos, psicológicos, sociológicos, metodológicos, los propios de la estructura funcional del deporte, etc.) sirve para actuar y para resolver situaciones de entrenamiento; igualmente, es capaz de interpretar en el momento de actuar en el entrenamiento o en la competición. Este tipo de conocimiento es relativo y no se puede generalizar, cada situación de entrenamiento no es válida para otras situaciones o contextos. Es un conocimiento que surge de la acción, que se va construyendo en el día a día y en las complejas situaciones contextualizadas.

Turpin (1998) nos dice que el entrenador ha de poseer una serie de conocimientos para afrontar con garantías el diseño y dirección del proceso de entrenamiento y de entre ellas nos conviene señalar la que hace mención a la evaluación del trabajo efectuado, la estimación de los procesos llevados a cabo y los progresos realizados.

Éste, como hemos dicho con anterioridad responde a nuestro objetivo a la hora de crear una herramienta de evaluación de la conducta técnico-táctica del jugador en el juego colectivo básico.

Dicha herramienta es un test de juego de 2vs2 que nos permitirá estudiar la intervención del jugador atendiendo a diferentes aspectos o facetas, pero que en este trabajo, nos va a servir para centrarnos en el desenvolvimiento técnico-táctico, en la adecuación, eficacia y eficiencia de las habilidades técnico-tácticas de los jugador en función de la situación de juego en que nos encontremos.

\section{Metodología de investigación.}

\subsection{Herramienta de recogida de datos: Test de juego 2 vs 2 .}

La herramienta elegida para la recogida de datos es un test de juego de 2vs2, con el cual pretendemos conocer, por un lado, el desenvolvimiento de los jugadores en una situación real, pero a la vez, y con mayor interés queremos analizar el conocimiento de los jugadores acerca de los principios básicos del deporte del fútbol.

\subsection{Explicación de test de juego de 2 vs 2 - procedimiento.}

- El test de 2v2 se desarrollará en un terreno de juego de 10x10 m., delimitado por líneas y conos en cada una de las esquinas.

- El saque de centro se realizará mediante un bote neutral.

- El juego será libre y habrá de respetar las reglas del fútbol-7, a excepción de la del fuera de juego, la cual no existirá.

- El saque de banda se realizará con el pie, al igual que el de fondo o tras tanto.

- El objetivo es conseguir el mayor número de tantos. Se considerará tanto cuando un jugador pare el balón (parada total ${ }^{3}$ ) tras la línea de fondo del otro equipo previo pase de un compañero. Por tanto, no se considerará gol cuando un jugador, tras realizar una conducción para el balón tras la citada línea.

- Se colocarán cuatro jugadores (círculos amarillos en el gráfico) alrededor del campo (uno en cada esquina) con el objetivo de que no se pierda tiempo si un balón sale del campo. Cada uno de estos jugadores tendrá un balón, que echará al jugador que haya de reanudar el juego.

- No hay portero, ni ningún jugador podrá asumir dicho rol. Por lo tanto, en ningún caso se podrá jugar con las manos (Gráfico 1).

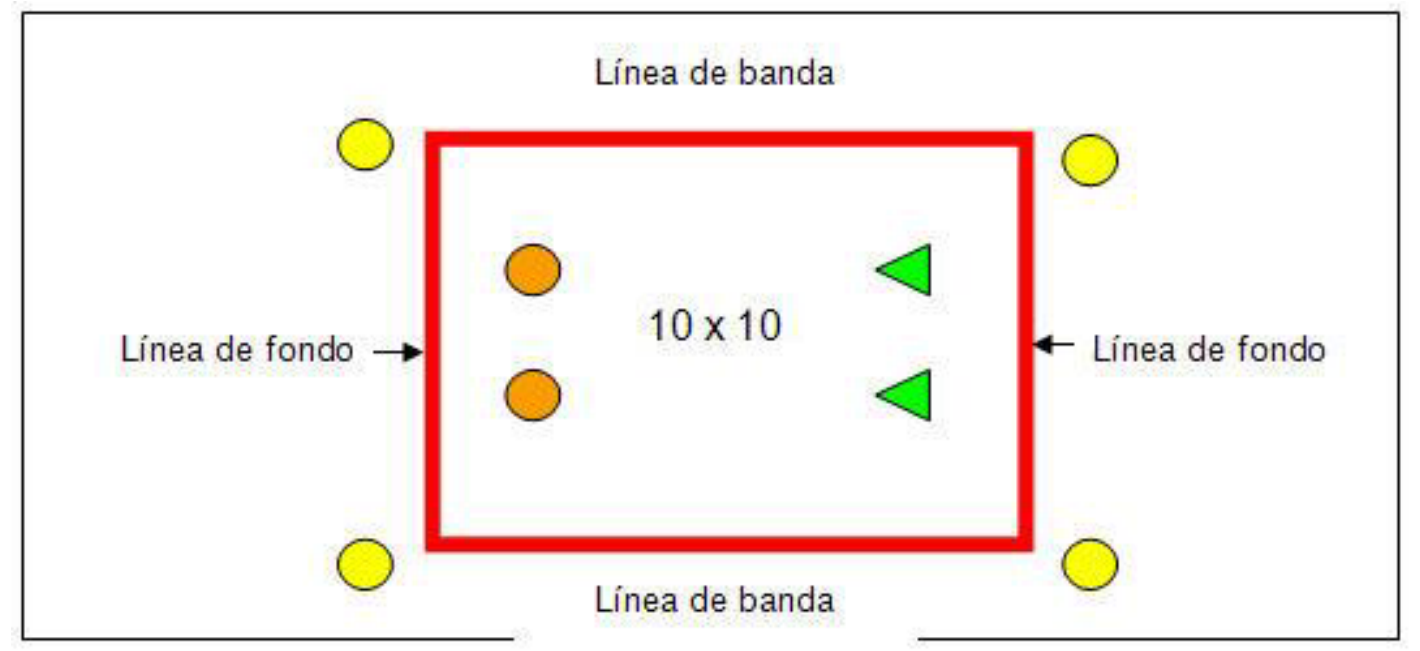




\section{Análisis del test}

\subsection{Definición de las categorías.}

Las categorías establecidas en el presente estudio han tomado como base inicial los trabajos de Ardá y Anguera (1999), los cuales se dedican al estudio de la acción ofensiva en fútbol-7. Básicamente, hemos seguido las fases del juego que dichos autores sugieren y hemos añadido la de desarrollo defensivo, la cual, debido a la naturaleza de aquel trabajo no aparecía en el mismo. Del mismo modo, las subcategorías las hemos redefinido en algunos casos y añadido en la mayoría, atendiendo, como ya hemos nombrado, a las características específicas de nuestra investigación.

En primer lugar hemos de decir que la utilización de un 2vs2 responde a los siguientes criterios:

- Posibilidad de analizar la actuación del jugador en la fase ofensiva y defensiva.

- Aparición de todos los fundamentos individuales y colectivos grupales del juego, a excepción de los específicos del portero.

- Desempeño de todos los roles por los que puede pasar el jugador:

* Poseedor del balón.

* Compañero del poseedor del balón.

* Oponente directo del poseedor de balón.

* Oponente directo de jugador no poseedor de balón, o oponente indirecto del poseedor de balón.

Definidos los anteriores criterios, diremos que los ejes vertebradotes en los que se basará el análisis del test que nos ocupa son:

- Rol del jugador ${ }^{4}$.

- Fundamento del juego utilizado.

- Conveniencia técnico-táctica.

- Corrección ejecutiva.

Conocidos los anteriores aspectos diremos que las categorías a emplear serán las siguientes (tabla 1 )

Cada una de las subcategorías que nos encontramos con anterioridad tendrán, en el presente estudio, una definición dicotómica, determinada por su corrección o incorrección técnica, por un lado, y por su conveniencia o inconveniencia técnico-táctica, o lo que, entre otros, Sampedro (1999) llama intención táctica. En este sentido Moreno y cols. (2000)nos viene a decir que «las intenciones tácticas coinciden con los objetivos de la acción, mientras que las respuestas técnicas son las elegidas o elaboradas como solución en los programas de acción».

En resumidas cuentas, en el test de 2vs2 atenderemos a los dos aspectos que a continuación tratamos de definir:

Corrección técnica de los fundamentos del juego: en este apartado atenderemos sólo al mecanismo ejecutivo del proceso de realización de la acción empleada, señalando su corrección o incorrección.

Conveniencia técnico-táctica (intención táctica): lo que perseguimos con la inclusión del análisis de este aspecto es comprobar si la ejecución de la acción va precedida de un análisis de la acción de juego, y, claro está, establecer diferencias entre los jugadores formados bajo una u otra metodología.

Una vez hecha la anterior aclaración, diremos que entenderemos que la intención del jugador será correcta cuando la acción ejecutada sea la más oportuna en la acción en que se encuentre, independientemente de la corrección ejecutiva de la misma. De este modo, nos vamos a encontrar con acciones correctas desde el punto de vista ejecutivo y técnico, pero incorrectas si nos referimos a su intención técnico-táctica o conveniencia en base a los principios del juego y viceversa, esto es, acciones ejecutadas incorrectamente pero cuya intención técnico-táctica era adecuada.

Podremos determinar así los siguientes aspectos:

- Capacidad de análisis del jugador.

Conocimiento del juego.

- Dominio de las habilidades específicas.

3.2. Evaluación de la intervención en el juego. Índice de actuación técnico-táctica.

El objetivo último del presente trabajo es, como ya dijimos en un principio, proporcionar a los técnicos una herramienta que les permita conocer con veracidad cual está siendo la actuación en el juego de sus jugadores.

Por ello pretendemos obtener un índice, es decir, reducir la actuación del jugador a un guarismo que nos orienta acerca de su desenvolvimiento en el juego.

A través de la revisión bibliográfica realizada, hemos encontrado índices de diferentes ámbitos, como pueden ser el de velocidad en el juego (Pino, 1999) o el de Garganta (2003), el cual intenta determinar la "perfomance en el juego» a través del análisis de diferentes aspectos del juego, tales como el desenvolvimiento técnico, las decisiones tomadas y las acciones de apoyo realizadas. Nuestra intención apunta hacia la obtención de un modo de actuar de fácil acceso para el técnico y es por ello que nos centramos en la utilización de un índice de acuerdos y desacuerdos.

Dicho índice se obtiene a través de la siguiente fórmula (gráfico 2):

\begin{tabular}{|c|}
\hline ACIERTOS \\
\hline ACIERTOS + ERRORES \\
\hline
\end{tabular}

El guarismo resultante será un índice entre 0 y 1 , donde el 1 corresponde al mayor índice de acierto y, por ende, el 0 al mayor índice de error.

A través de esteíndice intentamos cuantitativizar la valoración de la actuación del jugador, con el objetivo de hacerla más palpable y manipulable en su estudio. De esta manera la actuación del jugador será más susceptible de comparación entre jugadores o grupos de los mismos.

Tabla 1. Categorias de observación en el test de $2 v 2$

\begin{tabular}{|c|c|c|}
\hline $\begin{array}{l}\text { Inicio de la acción ofensiva } \\
\text { a. Poner el balón en juego: (Jugador }\end{array}$ & $\begin{array}{l}\text { Construccioin v desarrollo ofensivo } \\
\text { c. Juggador con bakin (jub) }\end{array}$ & $\begin{array}{c}\text { Desar mollo defensivo } \\
\text { e. Oponente directs del poscodor de }\end{array}$ \\
\hline a.1. saque después de recibir & $\begin{array}{l}\text { c. 1. Recepenon-control (Rec) } \\
\text { c. } 2 \text {. Conducción. (Cnd) }\end{array}$ & c.1. Acoso o pressing (Pre) \\
\hline $\tan$ o(saq) & c.3. Pase (Pas) & e.2. Entrada(Ent) \\
\hline a.2. saque de banda (saq) & c.4. Dribling.(Dri) & e.3. Tenporización.(Tpr) \\
\hline a.3. saque de falta (saq) & $\begin{array}{l}\text { c.5. Finta_Fib) } \\
\text { c.6. Carga(Cub) }\end{array}$ & e.4. Carga(Car) \\
\hline $\begin{array}{l}\text { b. Recuperar el baka: (Jugador sin } \\
\text { babon(odp) (oip)) } \\
\text { b. I. interceptación. (int) } \\
\text { b.2 robo.(rob) }\end{array}$ & $\begin{array}{l}\text { d. Conpaifa el poseador del balón. } \\
\text { (cpb) } \\
\text { d.1. Desmarque.(Dnnq) } \\
\text { d.2. Apoyo(Apo) } \\
\text { d.3. Finto.(Fin) }\end{array}$ & $\begin{array}{l}\text { f. Oponente indirecto del poseedor } \\
\text { de balón - oponente directo de } \\
\text { jugador sin babon. (oip) } \\
\text { f.1. Marcaje (Mje) } \\
\text { f.2. Cobertura (Cob) }\end{array}$ \\
\hline
\end{tabular}

'En el tes que nos ocupa el saque de banda se ralizarí con el pie. 


\subsection{Determinación de la observación.}

- Hemos desarrollado una observación sistemática, que intenta agrupar información a partir de criterios fijados previamente. Para su realización hemos realizado la categorización que anteriormente hemos introducido.

- La observación es natural, pues los hechos no son modificados por la presencia del observador y son grabados y posteriormente analizados tal como se suceden en la realización del test de juego.

- La observación no es participante, pues el observador no interacciona con los ejecutantes del test de forma que puedan variar su intervención. La única relación con lo mismo consiste en la explicación del test.

\subsubsection{Proceso de observación sistemática.}

\section{a) Técnicas de Recogida de datos.}

\section{a.1. Grabación.}

Los test de juego de 2vs2 han sido grabados para su posterior análisis mediante una grabación de vídeo. El instrumento con el cual lo hemos realizado ha sido una videocámara digital Thomson DVCVMD2S.

La videocámara se situaba a una distancia tal que permitiera recoger toda la extensión del terreno de juego en el cual habría de desarrollarse el test. Para ello nos ayudábamos de un trípode que permitiera gran estabilidada la videocámara.

\section{a.2. Agrupamiento de los alumnos.}

Los alumnos fueron agrupados de forma aleatoria, tanto con su compañero, como con sus adversarios. Dicho agrupamiento se respetó en la primera y segunda toma, con el fin de eliminar variables contaminantes procedentes del cambio de compañero o adversario en la realización del test. En los casos en los cuales no se pudo respetar este hecho se consideró el test como nulo.

\section{a.3. Identificadores de los jugadores.}

Los jugadores realizaban el test enfundados en unos petos de diferente color cada equipo, facilitando así la labor de observación y análisis de los mismos.

\section{b) Técnicas de Registro de datos.}

Una vez realizada la grabación de los test de juego procedemos al análisis de estos.

Los datos se extraían a partir de la observación sistemática a que hemos hecho referencia y mediante una hoja de registro de acontecimientos $^{5}$, en la cual recogíamos la frecuencia con que aparecían las conductas estipuladas en las diferentes categorías a que atendemos.
Cuando a lo largo de la observación han ido apareciendo las diferentes conductas objeto de la investigación se han ido anotando en el casillero oportuno en la hoja de registro.

\section{b.1. Fiabilidad y entrenamiento de observadores.}

La observación fue llevada a cabo por un observador, que previo al análisis de los test de juego de 2vs2 realizó un proceso de entrenamiento consistente en la visualización de varias tandas de test.

Se consideró que el observador tenía una fiabilidad óptima cuando sus registros coincidieron en más de un $90 \%$ al analizar 10 veces el mismo test.

\subsection{Muestra.}

La muestra analizada la forman un total de 59 sujetos de categoría alevín, de los cuales han sido analizadas 1342 habilidades técnico-tácticas mediante la hoja de registro de acontecimientos que con anterioridad recogimos.

4. Análisis de los resultados.

Los datos que vamos a extraer son:

* Frecuencia de habilidades técnico-tácticas realizadas e índice de corrección de cada una de ellas.

* Frecuencia de fases del juego e índice de corrección de cada fase.

* Índice de Corrección ejecutiva.

* Índice de Corrección decisional.

* Índice de Corrección técnico-táctica.

\subsection{Análisis de las habilidades técnico-tácticas utilizadas.}

En la tabla 2 se muestra la frecuencia de utilización de las habilidades técnico-tácticas.

Podemos observar en la tabla anterior como las habilidades técnico-tácticas más utilizadas son el pase y el control o recepción, las cuales aparecen de manera destacada respecto a las otras. Dichas habilidades son complementarias, pues no podemos entender que un pase ha alcanzado su objetivo si no conlleva una recepción y control del balón por parte de su compañero.

Este dato nos habla del carácter eminentemente colectivo que los jugadores han querido otorgar a su juego, pues otra de las habilidades que más destaca es la de apoyo.

Siguiendo con el análisis de las habilidades técnico-tácticas empleadas en el test de juego de 2vs2, vamos a centrarnos ahora en la corrección de dichas habilidades (tabla 3). Podemos comprobar como en la mayoría de los casos, el índice de corrección táctica se encuentra por encima del técnico, lo cual nos da una idea de que los jugadores eligen en un porcentaje elevado de las ocasiones la decisión correcta.

Analizando los resultados, podemos comprobar como los índices de corrección más altos corresponde al pase y al control del balón, lo cual corrobora los que anteriormente comentábamos del carácter colectivo que los jugadores han pretendido dar a su juego.

Tabla 2 Frecuencia de utilización de las habilidades téenico-tácticas

\begin{tabular}{cccc}
\hline Habilidad técnico-táctica & Frecuencia & Habilidad técrico-tactica & Frecuencia \\
Pase & 206 & Conducción & 75 \\
Control & 187 & Interceptación & 52 \\
Acoso & 141 & Robo & 42 \\
Apovo & 137 & Entraúa & 33 \\
Marcaic & 103 & Anticioacion & 9 \\
Desmarcue & 89 & Vzailancia & 9 \\
Saauc & 87 & Temporización & 6 \\
Regate & 85 & Carga & 3 \\
Cobertura & 77 & Finta & 1 \\
\hline
\end{tabular}


Tabla 3. Indices de correccionn de las habilidades técnico tácticas

\begin{tabular}{|c|c|c|c|c|c|c|c|c|}
\hline \multirow{3}{*}{$\begin{array}{l}\text { Habilidades téenico-tácticas } \\
\text { Sacue }\end{array}$} & \multicolumn{4}{|c|}{ Asnecto técnico } & \multicolumn{4}{|c|}{ Asrecto túctico } \\
\hline & \multicolumn{2}{|c|}{ correcto } & \multicolumn{2}{|c|}{ incorrecto } & \multicolumn{2}{|c|}{ correcto } & \multicolumn{2}{|c|}{ incorrecto } \\
\hline & 63 & $72.4 \%$ & 24 & $27.6 \%$ & 66 & $75 \% \%$ & 21 & $24.1 \%$ \\
\hline Irtercextación & 34 & $65.4 \%$ & 18 & $34.6 \%$ & 49 & $94.2 \%$ & 3 & $5.8 \%$ \\
\hline Robo & 42 & $100,0 \%$ & & & 42 & $100,0 \%$ & & \\
\hline A mticipación & 7 & $77.8 \%$ & 2 & $22.2 \%$ & 7 & $77.8 \%$ & 2 & $222 \%$ \\
\hline Conduceion & 60 & $80.0 \%$ & 15 & $20.0 \%$ & 53 & $70.7 \%$ & 22 & $293 \%$ \\
\hline Pas & 122 & $59,2 \%$ & 84 & $40,8 \%$ & 165 & $80,1 \%$ & 41 & $19,9 \%$ \\
\hline Regate & 33 & $38.8 \%$ & 52 & $61.2 \%$ & 14 & $16.5 \%$ & 71 & $83.5 \%$ \\
\hline Finta & & & 1 & $100.0 \%$ & & & 1 & $1000 \%$ \\
\hline Contwol & 150 & $80.2 \%$ & 37 & $19.8 \%$ & 162 & $86.6 \%$ & 25 & $13.4 \%$ \\
\hline Desmarque & 52 & $58.4 \%$ & 37 & $41.6 \%$ & 60 & $67.4 \%$ & 29 & $32.6 \%$ \\
\hline Apovo & 74 & $54.0 \%$ & 63 & $46.0 \%$ & 83 & $60.6 \%$ & 54 & $39.4 \%$ \\
\hline Acoso & 78 & $55,3 \%$ & 63 & $44.7 \%$ & 86 & $61,0 \%$ & 55 & $39,0 \%$ \\
\hline Entrada & 22 & $66.7 \%$ & 11 & $333 \%$ & 21 & $63.6 \%$ & 12 & $36.4 \%$ \\
\hline Carga & 3 & $100.0 \%$ & & & 3 & $100.0 \%$ & & \\
\hline Temporización & 4 & $66.7 \%$ & 2 & $33.3 \%$ & 4 & $66.7 \%$ & 2 & $33.3 \%$ \\
\hline Marcaic & 14 & $13.6 \%$ & 89 & $86.4 \%$ & 14 & $13.6 \%$ & 89 & $86.4 \%$ \\
\hline Vigilancia & 6 & $66.7 \%$ & 3 & $33.3 \%$ & 6 & $66.7 \%$ & 3 & $333 \%$ \\
\hline Cobertura & 47 & $61.0 \%$ & 30 & $39.0 \%$ & 63 & $81.8 \%$ & 14 & $182 \%$ \\
\hline
\end{tabular}

En el polo opuesto nos encontramos el regate, donde solo el $36 \%$ de los casos ha sido ejecutado correctamente y sólo en un $18 \%$ se ha hecho con una correcta adecuación técnico-táctica. Aquí nos encontramos un importante dato que nos informa de la necesidad que hay de tratar esas habilidades técnico-tácticas con los jugadores, haciéndoles más conocedores de la realidad del juego e intentando que comprendan de lo arriesgado de la habilidad del regate, pues supone una alta probabilidad de pérdida del balón. En este sentido tendríamos que analizar si el regate se ha hecho por propia iniciativa del poseedor del balón o por falta de apoyo de su compañero, con lo cual debería ser éste un dato en el cual habríamos de profundizar para así poner solución al mismo.

La otra habilidad donde el error a nivel técnico-táctico es muy elevado es en el marcaje. Nos denota esto una necesidad de trabajar contenidos que permitan y enseñen al alumno a centrar su atención en aspectos que no se circunscriban exclusivamente al balón, ya que éste es el principal problema a la hora de fallar en el marcaje. En este sentido el trabajo de Pino y cols. (1999) nos viene a destacar los principales errores que nos encontramos en los marcajes de los jugadores, siéndonos útil para trabajar y paliar esta carencia.

Los anteriores gráficos nos sirven como continuación del análisis realizado a raíz de los porcentajes de acierto y error en las diferentes habilidades y hacen más clara la visualización de estos aspectos.
Es muy importante destacar los casos de las habilidades de conducción y la entrada, pues nos dan una idea de realizar estudios o evaluaciones de la naturaleza de la que estamos realizando.

Podemos comprobar como en ambos casos el índice de corrección técnica supera al de corrección táctica y, por ende, al de corrección técnico-táctica. En una evaluación tradicional, en la cual sólo se prestaba atención a la ejecución técnica, el entrenador podría sacar la conclusión de que el jugador domina dichas habilidades, pues presenta unos índices de $0^{\prime} 8$ en la conducción y de 0,67 en la entrada (tabla 4). Pero al analizar el aspecto táctico podemos comprobar que aunque el jugador, o jugadores en este caso, saben ejecutar una conducción bastante bien, no eligen el momento adecuado para realizarla, lo mismo que ocurre con la entrada, pues en el momento en que decidieron realizar dichas acciones habría otras soluciones más adecuadas para resolver la situación de juego en que se encontraba.

Este hecho es el que queremos recalcar como la principal necesidad de la evaluación en el fútbol, saber no sólo si el jugador sabe ejecutar bien una acción, sino si lo hace de manera coherente al momento del juego.

\subsection{Análisis de actuación en diferentes fases del juego.}

Como podemos comprobar en el gráfico 3, la fase del juego más

Tabla 4, Indice de corrección por habilidades

\begin{tabular}{|c|c|c|c|c|}
\hline Habilidad & Fruxuencia & ICTE & ICTA & ICTT \\
\hline Saque & 87 & 0.72 & 0.76 & 0.74 \\
\hline Intercataxión & $\$ 2$ & 0.65 & 094 & 0.80 \\
\hline Robo & 42 & 1 & 1 & 1 \\
\hline Anticipacson & 9 & 0,78 & 0,78 & $0, \pi$ \\
\hline Conducción & 75 & 0.8 & 0.71 & 0.75 \\
\hline Pase & 206 & 0.59 & 0.80 & 0.20 \\
\hline Regnte & 85 & 0.39 & a. 16 & 0.28 \\
\hline Finta & 1 & 1 & 1 & 1 \\
\hline Control & 187 & 0.80 & 0.87 & 0.83 \\
\hline Desmarque & 89 & 0,58 & 0,67 & 0,63 \\
\hline Apovo & 137 & 0.54 & Q61 & 0.57 \\
\hline Acoso & 141 & 0.55 & 0,61 & 0.58 \\
\hline Entrata & 33 & 0.67 & 064 & 0.65 \\
\hline Carna & 3 & 1 & 1 & 1 \\
\hline Temporizacion & 6 & 0.67 & 067 & 0.67 \\
\hline Marcie & 103 & 0.14 & 0.14 & 0.14 \\
\hline Vigilancis & 9 & 0.67 & 0,67 & 0.67 \\
\hline Cobertura & 77 & 0.51 & 0.82 & 0.71 \\
\hline
\end{tabular}




\section{DISTRIBUCIÓN DE LAS HABILIDADES POR FASES DEL JUEGO}

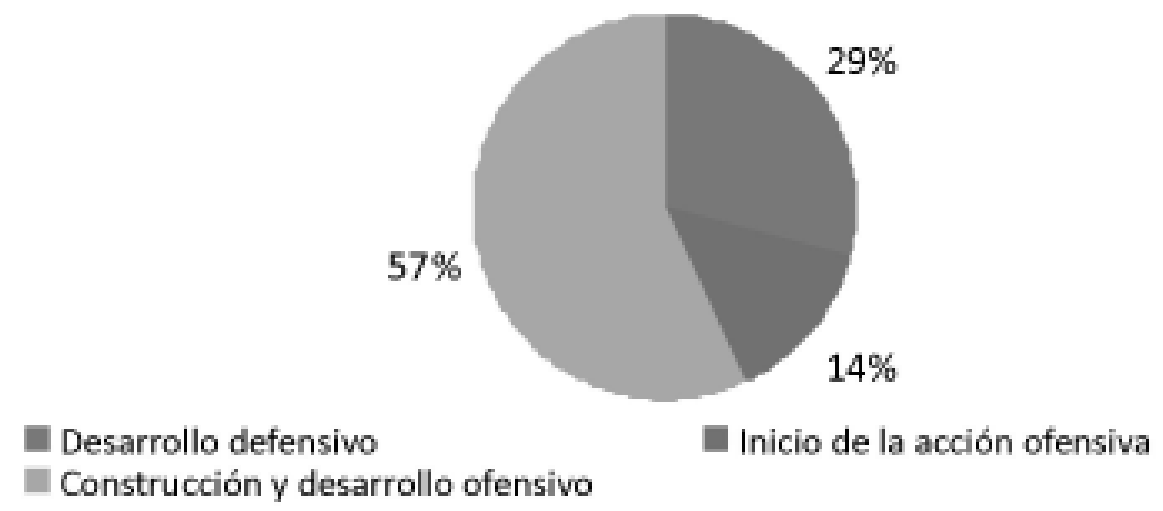

empleada por los jugadores es la de construcción y desarrollo ofensivo, mientras que la menos utilizada es la referida al inicio de la acción ofensiva.

Como punto de partida del análisis de los datos que podemos realizar, nos llama la atención el hecho de que en el test que hemos empleado, el tanto por ciento de jugadores dedicados a la tarea de ataque es igual que aquellos otros que los que han de desempeñar labores defensivas. Aunque esto es extrapolable a un gran porcentaje de las situaciones de juego real, en la situación en que hemos estudiado a los jugadores ha de resultar bastante más significativo, pues la implicación en el juego en una situación de 2vs2 siempre es directa, por lo cual el desequilibrio entre las fases del juego de desarrollo defensivo y de construcción y desarrollo ofensivo nos parece un dato muy significativo, más si tenemos en cuenta que el número de habilidades técnico-tácticas observadas en ambas fases es el mismo (tabla 5).

Este hecho nos proporciona un dato muy importante para el técnico y es que los jugadores prestan más atención al desarrollo ofensivo del juego, realizando más acciones en esta fase.

$\mathrm{Al}$ analizar los test de juego hemos podido comprobar como la actitud de los jugadores en la fase de desarrollo defensivo es mucho más pasiva que en la ofensiva, lo cual viene a corroborar la afirmación lanzada en el párrafo anterior.

Nuevamente el análisis realizado nos da una muestra de la información que a través de un proceso sistemático de evaluación podemos obtener. En este caso, nos denota una necesidad de prestar más atención en el entrenamiento a la comprensión y desarrollo de la fase defensiva del juego.

Todo lo anteriormente comentado se ve corroborado por los datos que mostramos en en la tabla $n^{\circ} 5$, donde podemos comprobar que existe una diferencia abismal entre el número de habilidades empleadas en la fase de construcción y desarrollo ofensivo y aquellas correspondientes a la fase de desarrollo defensivo. El dato se vuelve aún más significativo cuando comprobamos que el porcentaje de corrección entre ambas fases varía de manera sustanciosa a favor de la primera de las fases citadas. Esto viene a confirmar la necesidad de que el técnico, en el grupo de jugadores al que nos estamos refiriendo, preste mayor atención a la fase defensiva del juego.

Los datos mostrados en el gráfico $\mathrm{n}^{\circ} 4$ resultan determinantes con respecto a las cuestiones que hemos venido comentando en las últimas páginas. Los resultados obtenidos nos confirman una vez más la nece- sidad de un trabajo más exhaustivo en la comprensión por parte de los jugadores de la fase de desarrollo defensivo, así como en le tratamiento de las habilidades técnico-tácticas correspondientes a esta fase.

\section{3. Índices de corrección por sujetos.}

En última instancia vamos a recoger unos datos que nos parecen significativos y que nos vienen a señalar los índices de corrección en cada uno de los sujetos estudiados. El hecho de contemplar este dato resulta de vital importancia para el técnico, pues, recordando que tomamos el entrenamiento como un proceso didáctico, no hemos de caer en el error de realizarúnicamente una evaluación grupal, sino que hemos de atender a las diferencias individuales de cada uno de nuestros jugadores. Es por ello que una evaluación individualizada de cada jugador será indispensable para conseguir una mejora colectiva de todo el grupo (tabla 6, 7 y 8).

No es nuestra intención extendernos en este momento sobre los datos que hemos recogido en las tablas y gráficos anteriores, simplemente hemos querido hacer más gráfico el objetivo que perseguíamos con la realización del presente trabajo y hacer ver al técnico deportivo en general, y al de fútbol en particular de la cantidad de datos importantes y significativos para nuestra labor que podemos manejar al realizar un proceso de evaluación sistemático y riguroso.

\section{Conclusiones.}

La realización de este trabajo viene a responder a la necesidad existente en el entrenamiento del fútbol de una mayor seriedad y rigurosidad en los planteamientos existentes en el entrenamiento del fútbol, en general, y de las categorías de iniciación en particular.

Trabajos como éste se hacen necesarios en el mundo del fútbol base, con el fin de ajustar más los procesos de entrenamiento a la realidad de sus practicantes, a sus necesidades, posibilidades y limitaciones.

Enúltima instancia, y lo que, a nuestro modo de ver, nos parece más importante, abordar los procesos de evaluación del fútbol desde una perspectiva más integradora del entrenamiento nos proporcionará, no sólo más información, sino datos mucho más significativos y relevantes acerca de la actuación de los jugadores en la situación real de juego.

Es indispensable afrontar la evaluación de la actuación de los jugadores desde la perspectiva técnico-táctica, y no sólo de la técnica o de la táctica por separado, pues como hemos visto a lo largo de este trabajo, la información que obtenemos resulta mucho más útil y real.

\begin{tabular}{cc}
\hline Tabla 5. Número de habilidades en cada fase del juego \\
\hline Fase & $\mathbf{N}^{\circ}$ habilidades \\
Inicio de la accion ofensiva & 190 \\
Construccion y desarrollo ofeusivo & 769 \\
\hline Desarrollo defansino & 383 \\
\hline
\end{tabular}




\section{Índice de actuación por fases del juego}

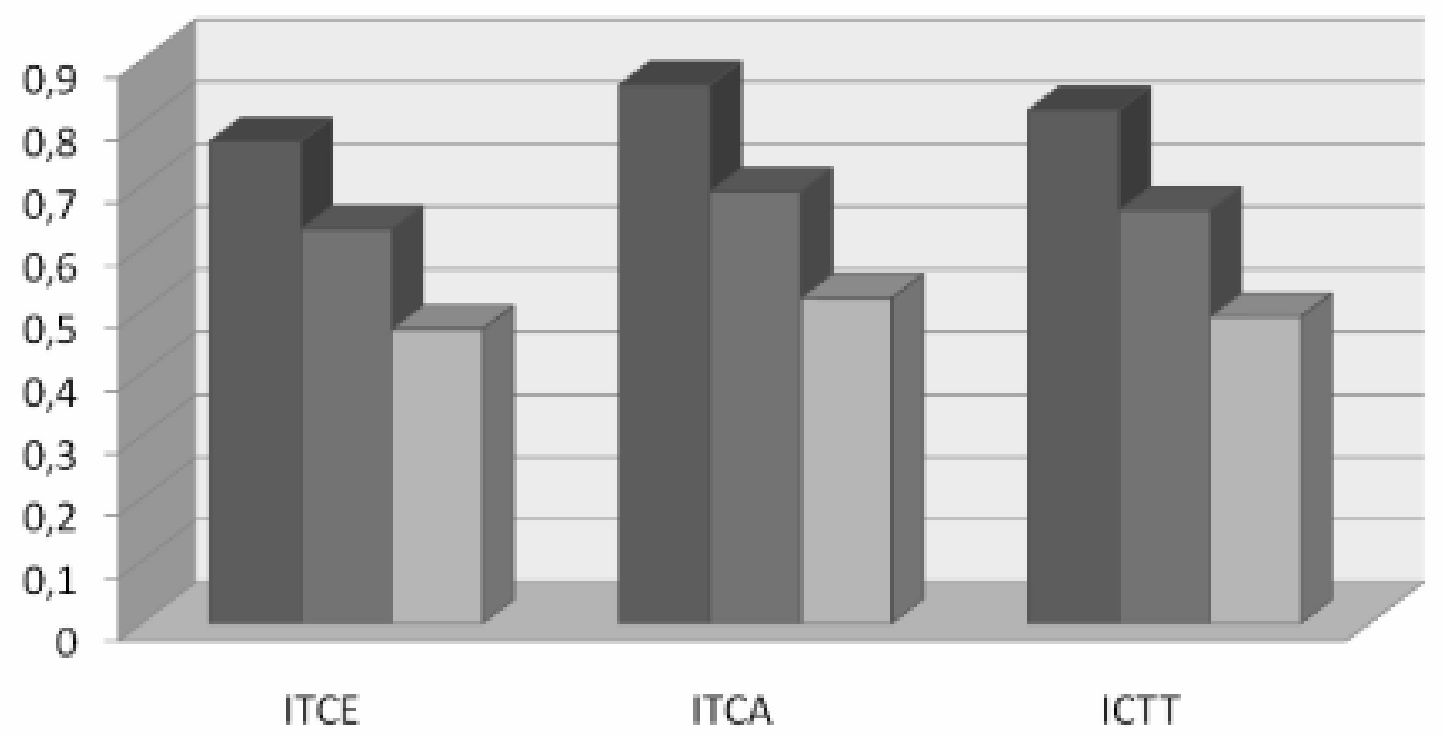

Inicio de la acción ofensiva $\square$ Construcción y desarrollo ofensivo $\square$ Desarrollo defensivo

Por último quisiéramos reseñar que en el presente trabajo hemos recogido sólo alguno de los datos que través del test realizado se pueden obtener, los cuales serán frutos de futuras investigaciones y de una tesis doctoral que en el momento se está realizando en torno a los procesos de formación y a la metodología de enseñanza en el fútbol base.

\section{Bibliografía.}

Anguera, Ma .T. (2003) Metodología básica de observación en Fútbol en: Ardá T. y Casal Sanjuro. Metodología de la enseñanza del Fútbol. Barcelona: Paidotribo.

Bruggemann, D. y Albrecht, D. (1993). Entrenamiento moderno del fútbol. Barcelona: Paidotribo.

Graca, A. y Olivera, J. (1997). La enseñanza de los juegos deportivos. Barcelona: Paidotribo..

Godik, M.A. y Popov, A.V.(1993). La preparación física del futbolista. Barcelona: Paidotribo.

Hernández Moreno, J. (1994). Fundamentos del deporte: análisis de las estructuras del juego deportivo. Barcelona: Paidotribo.

\begin{tabular}{cc}
\hline Tabla 6. Indice de corrección técnica por sujetes \\
\hline Masimo & 0.98 \\
Minimo & 0.32 \\
Media & 0.59 \\
Desviacion Tipica & 0.17 \\
\hline
\end{tabular}

\begin{tabular}{cc} 
Tabla 7. Indice de correceión tä́tica per sujet os \\
\hline Máximo & 0,95 \\
Minimo & 0.33 \\
Media & 0.66 \\
Desviación Tipion & 0.16 \\
\hline
\end{tabular}

\begin{tabular}{|c|c|}
\hline Máximo & 0,95 \\
\hline Minime & 0.36 \\
\hline Media & 0.63 \\
\hline Desviación Típica & 0,16 \\
\hline
\end{tabular}

Mombaerts, E. (1998). Fútbol. Entrenamiento y rendimiento colectivo. Barcelona: Hispano Europea.

Pascual, F. (1997). Planteamientos de entrenamiento físico-técnicotáctico globalizado en el fútbol actual. Training Fútbol, febrero, $\mathrm{n}^{\circ} 1$.

Pino, J. (1999). Desarrollo y aplicación de una metodología observacional para el análisis descriptivo de los medios técnico/ tácticos del juego de fútbol. Cáceres, Universidad de Extremadura, Tesis Doctoral inédita.

Pino, J. (1999). Evolución de los instrumentos y métodos de observación en fútbol. En Lecturas: Educación Física y Deportes. Revista Digital- $n^{\circ} 17$.

Romero, C.( 2000).- Hacia una concepción más integral del entrenamiento en el Fútbol. efdeportes.com (Buenos Aires) Año 5 (No 19), Marzo.

Sanz J.M. (1996). Diferencia entre la metodología del entrenamiento tradicional y el entrenamiento globalizado en el fútbol, Training Fútbol, abril, $\mathrm{n}^{\circ} 2$.

Seiru' lo, F. (1999). Criterios modernos de entrenamiento en el fútbol. Training Fútbol, noviembre, $\mathrm{n}^{\circ} 45$.

Turpin, B. (1998). Preparación y entrenamiento del futbolista. Barcelona. Hispano Europea.

\section{Notas}

${ }^{1}$ La Evaluación es «el proceso complejo de obtención de información y de su uso para formular juicios, que a su vez se utilizarán para tomar decisiones» (Tenbrik, 1984).

${ }^{2}$ Entendemos que la habilidad es la «capacidad adquirida por el aprendizaje de producir unos resultados previstos con el máximo de certeza y frecuentemente con el mínimo coste energético, o de ambas.» (knapp , 1981). No nos preocupe sólo si el jugador hace o no hace, sino si lo hace bien o no; no nos preocupa sólo si el jugador sabe pasar el balón (técnica), sino si lo hace en el momento adecuado, de manera eficaz y eficiente (habilidad técnico-táctica).

${ }^{3}$ Entendemos en este estudio por parada total cuando un jugador tras recibir el balón consigue detener la evolución y movimiento del mismo y se queda con su posesión.

${ }^{4}$ El jugador a lo largo del juego puede desempeñar cuatro roles:

Poseedor del balón. (jub), Compañero del poseedor del balón. (cpb), Oponente directo del poseedor del balón. (odp), Oponente indirecto del poseedor del balón -compañero del oponente directo del poseedor del balón -.(oip). Cada uno de dichos roles llevará implícitas actuaciones, encaminadas a la consecución de los objetivos y atendiendo a los principios del juego.

${ }^{5}$ Ver en anexo. 